\title{
Nuclear attenuation - 2 dimensional dependences at HERMES
}

\author{
Inti Lehmann ${ }^{* \dagger}$ \\ University of Glasgow, Scotland/UK \\ E-mail: i.lehmann@physics.gla.ac.uk
}

Hadron multiplicity ratios in semi-inclusive deep-inelastic scattering have been measured on neon, krypton and xenon targets relative to deuterium at an electron-beam energy of $27.6 \mathrm{GeV}$ by the HERMES experiment. They were determined as function of the virtual-photon energy $v$, its virtuality $Q^{2}$, the fractional hadron energy $z$ and the transverse hadron momentum with respect to the direction of the virtual photon $p_{t}$. Dependences are presented for positively and negatively charged pions and kaons as well as protons and antiprotons in a two-dimensional representation, i.e. in the form of detailed binning over one variable and three slices over the other variable. These results will help to constrain mechanisms and models of hadronization much more decisively than by the use of integrated results.

A few features are highlighted in this contribution showing, in particular, that the kinematic dependences of positively charged kaons and, in particular, protons deviate significantly from those of pions.

XVIII International Workshop on Deep-Inelastic Scattering and Related Subjects April 19 -23, 2010

Convitto della Calza, Firenze, Italy

\footnotetext{
${ }^{*}$ Speaker.

${ }^{\dagger}$ for the HERMES Collaboration
} 

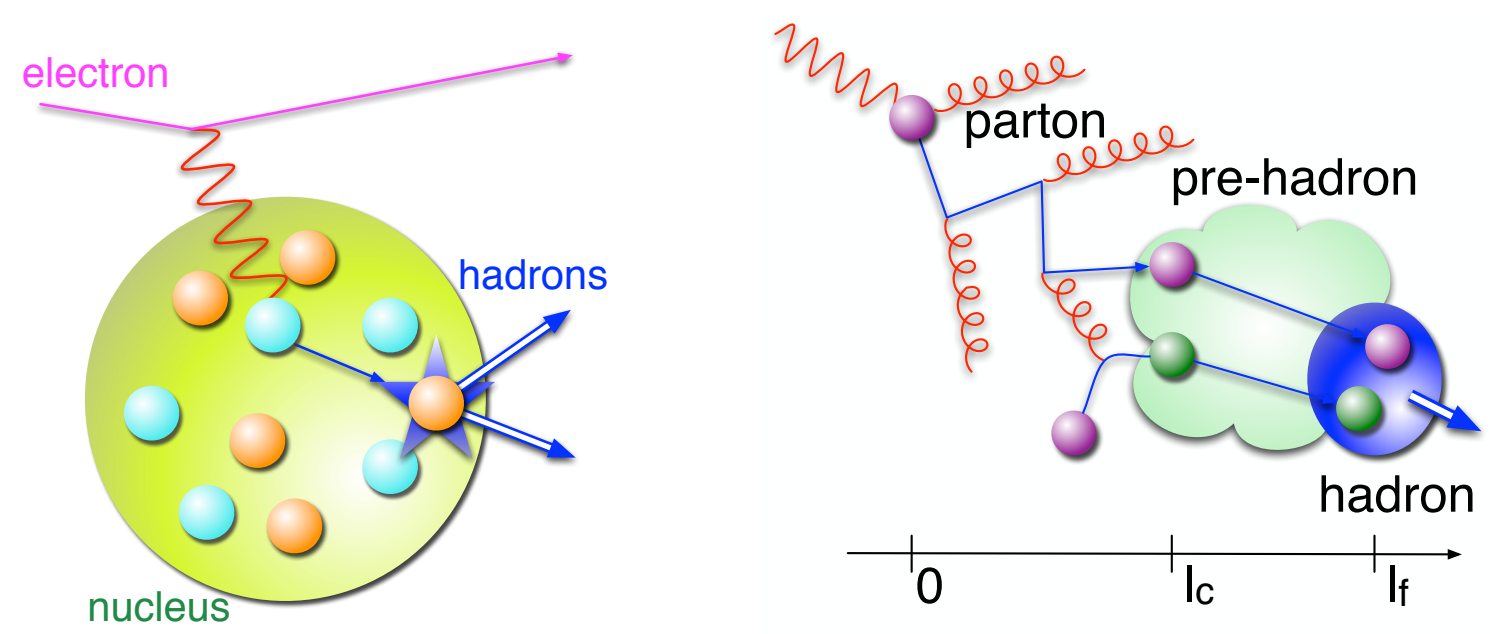

Figure 1: Schematic view of nuclear deep inelastic scattering and the hadronization process in the nucleus. In a first stage, at length scales below $l_{c}$, the parton is believed to propagate radiating gluons and undergoing partonic re-scattering. In this picture, the subsequently formed pre-hadron is thought to posses the quantum numbers of the hadron, be colourless but off its mass shell. The actual hadron is expected to be formed only after a propagation length $l_{f}$, which can be up to $10 \mathrm{fm}$ and, hence, outside or inside the nucleus depending on its size. Thus, using nuclei of different sizes, the properties of this process can be studied.

Semi-inclusive production of hadrons in deep-inelastic lepton nucleus scattering (SIDIS) provides a way to study quark fragmentation or hadronization. Naïve drawings depicting the principal concepts are sketched in Fig. 1. Lepto-production of hadrons has the virtue that the energy and momentum transfered to the hit parton are well determined, as it is "tagged" by the scattered lepton. In these studies the nucleus is basically used as a scale probe of the underlying hadronization mechanism: by using nuclei of increasing size one can investigate the the space(time) development of hadronization. The most convenient experimentally measurable observable for this process is the multiplicity ratio:

$$
R_{A}^{h}\left(v, Q^{2}, z, p_{t}^{2}\right)=\frac{\left(\frac{N^{h}\left(v, Q^{2}, z, p_{t}^{2}\right)}{N^{e}\left(v, Q^{2}\right)}\right)_{A}}{\left(\frac{N^{h}\left(v, Q^{2}, z, p_{t}^{2}\right)}{N^{e}\left(v, Q^{2}\right)}\right)_{D}},
$$

which is the ratio of the number of hadrons $N^{h}$ produced and the number of deep-inelastic scattering events $N^{e}$ on a given nucleus $A$ compared to the same ratio on the deuteron $D$. This ratio depends on four independent kinematic variables, which were taken to be the photon energy $v$ and its virtuality $Q^{2}$, the fraction of the virtual-photon energy carried by the hadron $z$, and the square of the hadron momentum component transverse to the direction of the virtual photon $p_{t}^{2}$.

The available experimental data [1-6] are presented in most cases as a function of one variable, integrating over the other variables within the experimental acceptance (one-dimensional dependences), in order to keep the statistical uncertainties at a reasonable level. Only in one case was a two-dimensional dependence extracted, for a combined sample of charged pions [6]. In this analysis the same data as that of Ref. [6] have been used to study $R_{A}^{h}$ for all charged hadron final states separately for neon $(\mathrm{Ne})$, krypton $(\mathrm{Kr})$, and xenon $(\mathrm{Xe})$ targets, using a detailed binning in 

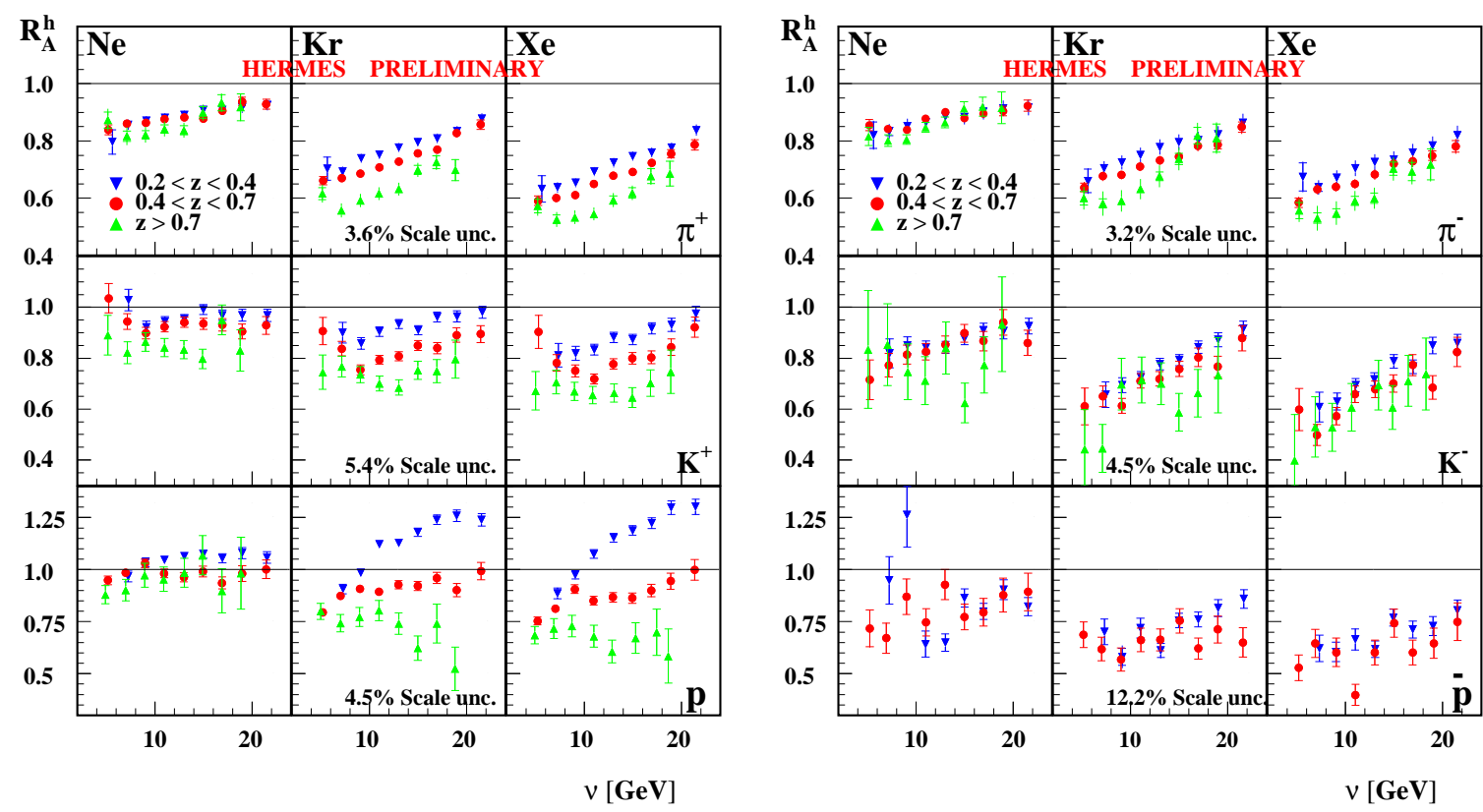

Figure 2: Dependence of $R_{A}^{h}$ on $v$ for positively and negatively charged hadrons for three slices in $z$ as indicated in the legend. The inner and outer error bars indicate the statistical and total uncertainties, respectively. For the latter the statistical and systematic bin-to-bin uncertainties were added in quadrature. The scale uncertainties depend only on the hadron type and are, hence, stated separately.

one variable and three slices in another one. This allows us to study the dependences in more detail, while keeping the statistical errors, at least for pions, $K^{+}$and protons, at moderate levels.

From the theoretical side there is significant interest in hadron-multiplicity ratios, as exemplified by the diversity of model calculations in Refs. [7-12] and references therein. It is beyond the scope of this report to compare the results of the various models with the data. Instead some of the most prominent features of the data are presented and discussed. It is expected that the present results provide the input needed to further constrain the models of hadronization.

The measurements were performed with the HERMES spectrometer [13] using $27.6 \mathrm{GeV}$ positron and electron beams stored in HERA at DESY. SIDIS data were collected in the years 1999, 2000, 2004 and 2005 with gaseous targets of D, Ne, Kr, and Xe. The identification of charged hadrons was accomplished using information from the dual-radiator ring-imaging Čerenkov detector (RICH) [14], which provides separation of pions, kaons and (anti)protons in the momentum range between 2 and $15 \mathrm{GeV} / \mathrm{c}$. The analysis was performed analogously to the analysis described in Ref. [6], using the same constraints with the sole exception of the hadron identification algorithm. Here we used an approach, which is based on direct ray tracing [15].

The multiplicity ratio $R_{A}^{h}$, as defined in Eq. 1 , for $\mathrm{Ne}, \mathrm{Kr}$, and Xe targets was determined for combinations of the kinematic variables $v, Q^{2}, z$, and $p_{t}^{2}$ for the six hadron types: $\pi^{+}, K^{+}, p$, $\pi^{-}, K^{-}, \bar{p}$, using fine binning in one of the variables, while using three course slices in another variable, and integrating over the other variables within the acceptance of the experiment. Here we only show a few examples that highlight the observed effects. A publication containing the full information is currently in preparation. 

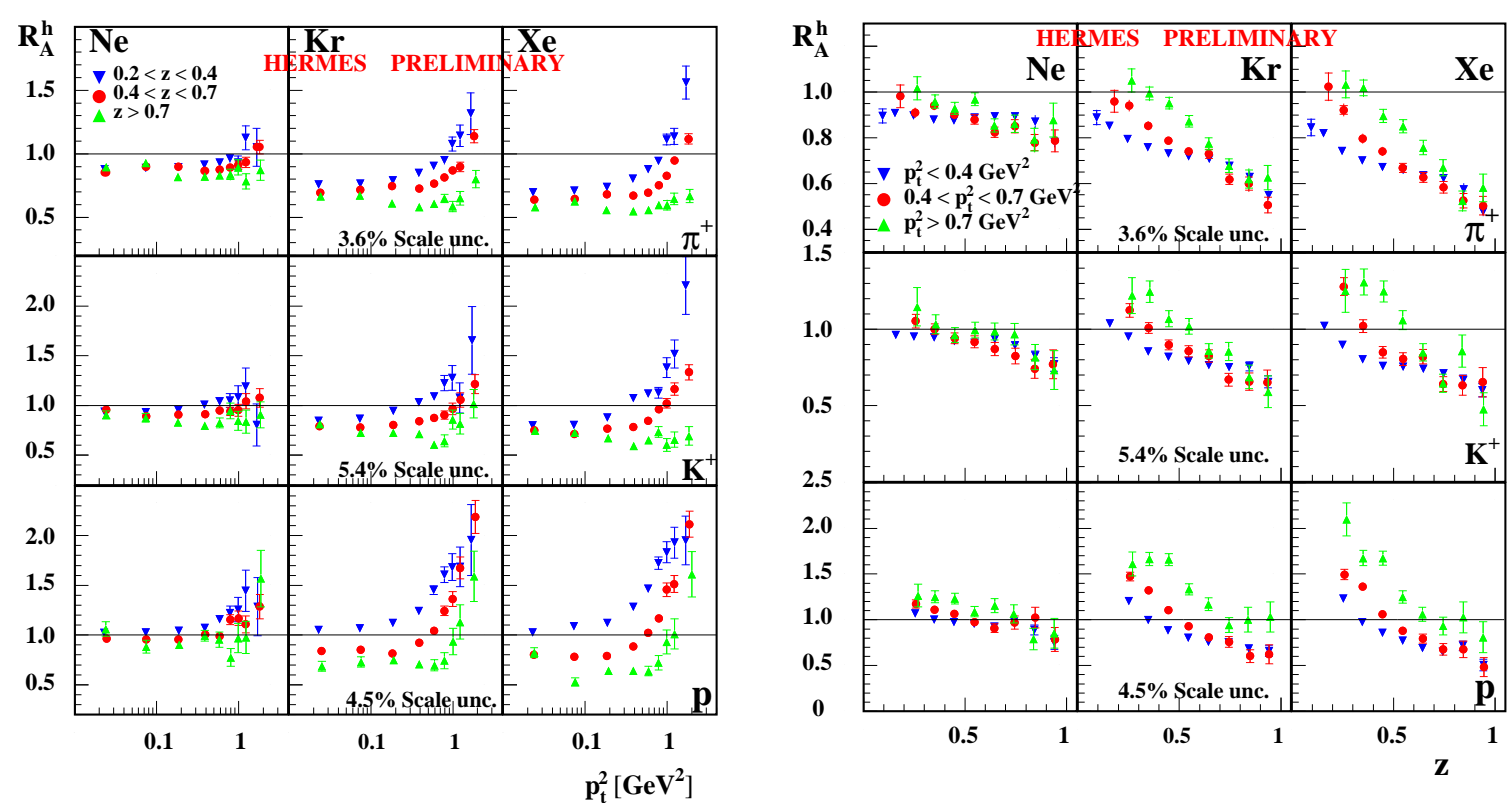

Figure 3: Dependences of $R_{A}^{h}$ for positively charged hadrons on $p_{t}^{2}$ for three slices over $z$ (left), and on $z$ for three slices over $p_{t}^{2}$ (right). Slices are taken as indicated in the respective legends. Statistical and total errors are shown as in the previous figure.

The dependence of $R_{A}^{h}$ on $v$ for three slices in $z$ is shown in Fig. 2. The global trend that $R_{A}^{h}$ steadily increases with $v$ seems to be broken at low values of $v$ for the higher $z$-ranges for $\pi^{+}, \pi^{-}$ and, in particular, for $K^{+}$and protons.

The results for protons are very different from those for the other hadrons. For the heavier nuclei, $R_{A}^{h}$ behaves very differently for the three $z$-slices, becoming even larger than unity at higher $v$ for the lower $z$ slice. In contrast, for $z>0.7, R_{A}^{h}$ is smaller than unity, and remains constant or drops with $v$. At the lowest values of $v$ the differences between the $z$ ranges seem to disappear. A possible explanation may be due to differing kinematic dependences of hadronization and knockout processes. In contrast to other hadrons, for protons the production may be dominated by the latter, at least in certain kinematic regimes. An enhanced yield, by factors of 3 to 10, of protons compared to antiprotons confirms this conjecture.

The left panel of Fig. 3 shows the dependence of $R_{A}^{h}$ on $p_{t}^{2}$ for three slices in $z$ for positively charged hadrons. For $\pi^{-}$a compatible behaviour within statistical errors compared to $\pi^{+}$was found. The rise at high $p_{t}^{2}$ is attributed to a broadening of the $p_{t}^{2}$ distribution. Such $p_{t}^{2}$-behaviour was predicted for values of $z \geq 0.5$ in Ref. [8]. However, the enhancement of $R_{A}^{h}$ at large values of $p_{t}^{2}$ predicted in Ref. [8] considerably overshoots our data, in particular for pions and kaons. Protons show a much stronger rise in the $p_{t}^{2}$ dependence which, in contrast to the case of pions and kaons, does hardly diminish when going to higher ranges in $z$. This is consistent with assuming a large contribution from knock out processes.

Another representation of the $z$-dependence of the broadening is given by plotting $R_{A}^{h}$ on $z$ for three slices in $p_{t}^{2}$ in the right panel of Fig. 3. This dependence of $R_{A}^{h}$ on $z$ turns out to be stronger at higher values of $p_{t}^{2}$, an effect which is emphasised with increasing target mass. At high $z$ the dependence on $p_{t}^{2}$ disappears, at least for $\pi^{+}, \pi^{-}$, and $K^{+}$. The effect for protons is similar, but 
much stronger (note the vertical scale).

In summary, we have extracted first two-dimensional multiplicity ratios for protons, antiprotons and positively and negatively charged pions and kaons separately. By giving this ratio for neon, krypton and xenon targets normalised to deuterium we observe significant structures which will help to constrain future models on hadronization. The $v$ dependence of positive kaons shows distinct features while $K^{-}$behave similar to both positive and negative pions; this is somehow reversed in the $z$ and $p_{t}^{2}$ dependences where positive kaons behave similarly to pions. A very striking observation is that the dependence of protons on $v$ changes its slope from a strong rise to a constant, if not a decrease, when looking at different slices of $z$. A possible interpretation of the latter effect may be that a large fraction of the observed protons could be produced in knock-out processes rather than hadronization.

We gratefully acknowledge the DESY management for its support, the staff at DESY and the collaborating institutions for their significant effort, and our national funding agencies for financial support.

\section{References}

[1] J. Ashman et al. [EMC], Z. Phys. C52 (1991) 1.

[2] M. Adams et al. [E665], Phys. Rev. D50 (1994) 1836.

[3] A. Airapetian et al. [HERMES], Eur. Phys. J. C20 (2001) 479.

[4] A. Airapetian et al. [HERMES], Phys. Lett. B577 (2003) 37.

[5] A. Airapetian et al. [HERMES], Phys. Rev. Lett. 96 (2006) 162301.

[6] A. Airapetian et al. [HERMES], Nucl. Phys. B780 (2007) 1.

[7] A. Accardi, Phys. Lett. B649 (2007) 384.

[8] B.Z. Kopeliovich, J. Nemchik, E. Predazzi and A. Hayashigaki, Nucl. Phys. A740 (2004) 211.

[9] T. Falter et al. , Phys. Lett. B594 (2004) 61; Phys. Rev. C70 (2004) 054609.

[10] N. Akopov, L. Grigoryan, Z. Akopov, Phys. Rev. C76 (2007) 065203.

[11] K. Gallmeister, U. Mosel, Nucl. Phyc. A 801 (2008) 68.

[12] R. Sassot, M. Stratmann and P. Zurita, arXiv:0906.5521 [hep-ph].

[13] K. Ackerstaff et al. [HERMES], NIM A417 (1998) 230.

[14] N. Akopov et al. NIM A479 (2002) 511.

[15] R. Lamb and A. Hillenbrand, HERMES Internal Note 07-017 (2008). 